\title{
Tighter monogamy relations in multipartite systems
}

\author{
Zhi-Xiang Jin * \\ School of Mathematical Sciences, Capital Normal University, \\ Beijing 100048, China \\ Jun $\mathrm{Li}^{\dagger}$ \\ School of Mathematical Sciences, Capital Normal University, \\ Beijing 100048, China \\ Tao $\mathrm{Li}^{\ddagger}$ \\ School of Science, Beijing Technology and Business University, \\ Beijing 100048, China \\ Shao-Ming Fei ${ }^{\S}$ \\ School of Mathematical Sciences, Capital Normal University, \\ Beijing 100048, China \\ Max-Planck-Institute for Mathematics in the Sciences, \\ Leipzig 04103, Germany
}

October 15, 2018

\begin{abstract}
Monogamy relations characterize the distributions of entanglement in multipartite systems. We investigate monogamy relations related to the concurrence $C$, the entanglement of formation $E$, negativity $N_{c}$ and Tsallis- $q$ entanglement $T_{q}$. Monogamy relations for the $\alpha$ th power of entanglement have been derived, which are tighter than the existing entanglement monogamy relations for some classes of quantum states. Detailed examples are presented.
\end{abstract}

\footnotetext{
*Corresponding author: jzxjinzhixiang@126.com

${ }^{\dagger}$ Corresponding author: lijunnl123@163.com

‡Corresponding author: litao@btbu.edu.cn

$\S$ Corresponding author: feishm@mail.cnu.edu.cn
} 


\section{INTRODUCTION}

Due to the essential roles played in quantum communication and quantum information processing, quantum entanglement [1, 2, 3, 4, 5, 6, 7, 8, has been the subject of many recent studies in recent years. The study of quantum entanglement from various viewpoints has been a very active area and has led to many impressive results. As one of the fundamental differences be tween quantumand classical correlations, an essential property of entanglement is that a quantum system entangled with one of the other subsystems limits its entanglement with the remaining ones. The monogamy relations give rise to the distribution of entanglement in the multipartite quantum systems.Moreover, themonogamy property has emerged as the ingredient in the security analysis of quantum key distribution 9 .

For a tripartite system $A, B$, and $C$, the usual monogamy of an entanglement measure $\mathcal{E}$ implies that [10] the entanglement between $A$ and $B C$ satisfies $\mathcal{E}_{A \mid B C} \geqslant \mathcal{E}_{A B}+\mathcal{E}_{A C}$. However, such monogamy relations are not always satisfied by all entanglement measures for all quantum states. In fact, it has been shown that the squared concurrence $C^{2}$ [11, 12] and entanglement of formation $E^{2}[13$ ] satisfy the monogamy relations for multiqubit states. The monogamy inequality was further generalized to various entanglement measures such as continuousvariable entanglement [14, 15, 16], squashed entanglement [10, 17, 18, entanglement negativity [19, 20, 21, 22, 23, Tsallis- $q$ entanglement [24, 25], and Renyi entanglement 26, 27, 28.

In this paper, we derive monogamy inequalities which are tighter than all the existing ones, in terms of the concurrence $C$, the entanglement of formation $E$, negativity $N_{c}$, and Tsallis- $q$ entanglement $T_{q}$.

\section{TIGHTER MONOGAMY RELATIONS FOR CONCURRENCE}

We first consider the monogamy inequalities satisfied by the concurrence. Let $\mathbb{H}_{X}$ denote a discrete finite-dimensional complex vector space associated with a quantum subsystem $X$. For a bipartite pure state $|\psi\rangle_{A B}$ in vector space $\mathbb{H}_{A} \otimes \mathbb{H}_{B}$, the concurrence is given by [29, 30, 31] $C\left(|\psi\rangle_{A B}\right)=\sqrt{2\left[1-\operatorname{Tr}\left(\rho_{A}^{2}\right)\right]}$, where $\rho_{A}$ is the reduced density matrix by tracing over the subsystem $B, \rho_{A}=$ $\operatorname{Tr}_{B}\left(|\psi\rangle_{A B}\langle\psi|\right)$. The concurrence for a bipartite mixed state $\rho_{A B}$ is defined by the convex roof extension $C\left(\rho_{A B}\right)=\min _{\left\{p_{i},\left|\psi_{i}\right\rangle\right\}} \sum_{i} p_{i} C\left(\left|\psi_{i}\right\rangle\right)$, where the minimum is taken over all possible decompositions of $\rho_{A B}=\sum_{i} p_{i}\left|\psi_{i}\right\rangle\left\langle\psi_{i}\right|$, with $p_{i} \geqslant 0, \sum_{i} p_{i}=1$ and $\left|\psi_{i}\right\rangle \in \mathbb{H}_{A} \otimes \mathbb{H}_{B}$.

For a tripartite state $|\psi\rangle_{A B C}$, the concurrence of assistance is defined by 32, 33.

$$
C_{a}\left(|\psi\rangle_{A B C}\right) \equiv C_{a}\left(\rho_{A B}\right)=\max _{\left\{p_{i},\left|\psi_{i}\right\rangle\right\}} \sum_{i} p_{i} C\left(\left|\psi_{i}\right\rangle\right)
$$


where the maximum is taken over all possible decompositions of $\rho_{A B}=$ $\operatorname{Tr}_{C}\left(|\psi\rangle_{A B C}\langle\psi|\right)=\sum_{i} p_{i}\left|\psi_{i}\right\rangle_{A B}\left\langle\psi_{i}\right|$. When $\rho_{A B}=|\psi\rangle_{A B}\langle\psi|$ is a pure state, one has $C\left(|\psi\rangle_{A B}\right)=C_{a}\left(\rho_{A B}\right)$.

For an $N$-qubit state $\rho_{A B_{1} \cdots B_{N-1}} \in \mathbb{H}_{A} \otimes \mathbb{H}_{B_{1}} \otimes \cdots \otimes \mathbb{H}_{B_{N-1}}$, the concurrence $C\left(\rho_{A \mid B_{1} \cdots B_{N-1}}\right)$ of the state $|\psi\rangle_{A \mid B_{1} \cdots B_{N-1}}$, viewed as a bipartite state under the partition $A$ and $B_{1}, B_{2}, \cdots, B_{N-1}$, satisfies 34 ]

$$
\begin{aligned}
& C^{\alpha}\left(\rho_{A \mid B_{1}, B_{2} \cdots, B_{N-1}}\right) \\
& \geqslant C^{\alpha}\left(\rho_{A B_{1}}\right)+C^{\alpha}\left(\rho_{A B_{2}}\right)+\cdots+C^{\alpha}\left(\rho_{A B_{N-1}}\right),
\end{aligned}
$$

for $\alpha \geqslant 2$, where $\rho_{A B_{i}}=\operatorname{Tr}_{B_{1} \cdots B_{i-1} B_{i+1} \cdots B_{N-1}}\left(\rho_{A B_{1} \cdots B_{N-1}}\right)$. The relation (1) is further improved so that for $\alpha \geqslant 2$, if $C\left(\rho_{A B_{i}}\right) \geqslant C\left(\rho_{A \mid B_{i+1} \cdots B_{N-1}}\right)$ for $i=$ $1,2, \cdots, m$ and $C\left(\rho_{A B_{j}}\right) \leqslant C\left(\rho_{A \mid B_{j+1} \cdots B_{N-1}}\right.$ for $j=m+1, \cdots, N-2, \forall 1 \leqslant$ $m \leqslant N-3, N \geqslant 4$, then [35],

$$
\begin{aligned}
& C^{\alpha}\left(\rho_{A \mid B_{1} B_{2} \cdots B_{N-1}}\right) \geqslant \\
& C^{\alpha}\left(\rho_{A B_{1}}\right)+\frac{\alpha}{2} C^{\alpha}\left(\rho_{A B_{2}}\right)+\cdots+\left(\frac{\alpha}{2}\right)^{m-1} C^{\alpha}\left(\rho_{A B_{m}}\right) \\
& +\left(\frac{\alpha}{2}\right)^{m+1}\left[C^{\alpha}\left(\rho_{A B_{m+1}}\right)+\cdots+C^{\alpha}\left(\rho_{A B_{N-2}}\right)\right] \\
& +\left(\frac{\alpha}{2}\right)^{m} C^{\alpha}\left(\rho_{A B_{N-1}}\right)
\end{aligned}
$$

and for all $\alpha<0$,

$$
\begin{aligned}
& C^{\alpha}\left(\rho_{A \mid B_{1} B_{2} \cdots B_{N-1}}\right)< \\
& K\left[C^{\alpha}\left(\rho_{A B_{1}}\right)+C^{\alpha}\left(\rho_{A B_{2}}\right)+\cdots+C^{\alpha}\left(\rho_{A B_{N-1}}\right)\right],
\end{aligned}
$$

where $K=\frac{1}{N-1}$.

In the following, we show that these monogamy inequalities satisfied by the concurrence can be further refined and become even tighter. For convenience, we denote $C_{A B_{i}}=C\left(\rho_{A B_{i}}\right)$ the concurrence of $\rho_{A B_{i}}$ and $C_{A \mid B_{1}, B_{2}, \cdots, B_{N-1}}=$ $C\left(\rho_{A \mid B_{1} \cdots B_{N-1}}\right)$. We first introduce two lemmas.

Lemma 1. For any real number $x$ and $t, 0 \leqslant t \leqslant 1, x \in[1, \infty]$, we have $(1+t)^{x} \geqslant 1+\left(2^{x}-1\right) t^{x}$.

Proof. Let $f(x, y)=(1+y)^{x}-y^{x}$ with $x \geqslant 1, y \geqslant 1$, then, $\frac{\partial f}{\partial y}=x[(1+$ $\left.y)^{x-1}-y^{x-1}\right] \geqslant 0$. Therefore, $f(x, y)$ is an increasing function of $y$, i.e., $f(x, y) \geqslant$ $f(x, 1)=2^{x}-1$. Set $y=\frac{1}{t}, 0<t \leqslant 1$, and we obtain $(1+t)^{x} \geqslant 1+\left(2^{x}-1\right) t^{x}$. When $t=0$, the inequality is trivial.

Lemma 2. For any $2 \otimes 2 \otimes 2^{n-2}$ mixed state $\rho \in \mathbb{H}_{A} \otimes \mathbb{H}_{B} \otimes \mathbb{H}_{C}$, if $C_{A B} \geqslant C_{A C}$, we have

$$
C_{A \mid B C}^{\alpha} \geqslant C_{A B}^{\alpha}+\left(2^{\frac{\alpha}{2}}-1\right) C_{A C}^{\alpha},
$$

for all $\alpha \geqslant 2$. 
Proof. It has been shown that $C_{A \mid B C}^{2} \geqslant C_{A B}^{2}+C_{A C}^{2}$ for arbitrary $2 \otimes 2 \otimes 2^{n-2}$ tripartite state $\rho_{A B C}\left[11,37\right.$. Then, if $C_{A B} \geqslant C_{A C}$, we have

$$
\begin{aligned}
C_{A \mid B C}^{\alpha} & \geqslant\left(C_{A B}^{2}+C_{A C}^{2}\right)^{\frac{\alpha}{2}} \\
& =C_{A B}^{\alpha}\left(1+\frac{C_{A C}^{2}}{C_{A B}^{2}}\right)^{\frac{\alpha}{2}} \\
& \geqslant C_{A B}^{\alpha}\left[1+\left(2^{\frac{\alpha}{2}}-1\right)\left(\frac{C_{A C}^{2}}{C_{A B}^{2}}\right)^{\frac{\alpha}{2}}\right] \\
& =C_{A B}^{\alpha}+\left(2^{\frac{\alpha}{2}}-1\right) C_{A C}^{\alpha}
\end{aligned}
$$

where the second inequality is due to Lemma 1 . As the subsystems $A$ and $B$ are equivalent in this case, we have assumed that $C_{A B} \geqslant C_{A C}$ without loss of generality. Moreover, if $C_{A B}=0$ we have $C_{A B}=C_{A C}=0$. That is to say the lower bound becomes trivially zero.

From Lemma 2, we have the following theorem.

Theorem 1. For an $N$-qubit mixed state, if $C_{A B_{i}} \geqslant C_{A \mid B_{i+1} \cdots B_{N-1}}$ for $i=$ $1,2, \cdots, m$, and $C_{A B_{j}} \leq C_{A \mid B_{j+1} \cdots B_{N-1}}$ for $j=m+1, \cdots, N-2, \forall 1 \leq m \leq$ $N-3, N \geqslant 4$, then we have

$$
\begin{aligned}
C_{A \mid B_{1} B_{2} \cdots B_{N-1}}^{\alpha} & \\
\geqslant & C_{A B_{1}}^{\alpha}+\left(2^{\frac{\alpha}{2}}-1\right) C_{A B_{2}}^{\alpha}+\cdots+\left(2^{\frac{\alpha}{2}}-1\right)^{m-1} C_{A B_{m}}^{\alpha} \\
& +\left(2^{\frac{\alpha}{2}}-1\right)^{m+1}\left(C_{A B_{m+1}}^{\alpha}+\cdots+C_{A B_{N-2}}^{\alpha}\right) \\
& +\left(2^{\frac{\alpha}{2}}-1\right)^{m} C_{A B_{N-1}}^{\alpha}
\end{aligned}
$$

for all $\alpha \geqslant 2$.

Proof. From the inequality (44), we have

$$
\begin{aligned}
& C_{A \mid B_{1} B_{2} \cdots B_{N-1}}^{\alpha} \\
& \geqslant C_{A B_{1}}^{\alpha}+\left(2^{\frac{\alpha}{2}}-1\right) C_{A \mid B_{2} \cdots B_{N-1}}^{\alpha} \\
& \geqslant C_{A B_{1}}^{\alpha}+\left(2^{\frac{\alpha}{2}}-1\right) C_{A B_{2}}^{\alpha}+\left(2^{\frac{\alpha}{2}}-1\right)^{2} C_{A \mid B_{3} \cdots B_{N-1}}^{\alpha} \\
& \geqslant \cdots \\
& \geqslant C_{A B_{1}}^{\alpha}+\left(2^{\frac{\alpha}{2}}-1\right) C_{A B_{2}}^{\alpha}+\cdots+\left(2^{\frac{\alpha}{2}}-1\right)^{m-1} C_{A B_{m}}^{\alpha} \\
& \quad+\left(2^{\frac{\alpha}{2}}-1\right)^{m} C_{A \mid B_{m+1} \cdots B_{N-1}}^{\alpha} .
\end{aligned}
$$

Similarly, as $C_{A B_{j}} \leqslant C_{A \mid B_{j+1} \cdots B_{N-1}}$ for $j=m+1, \cdots, N-2$, we get

$$
\begin{aligned}
C_{A \mid B_{m+1} \cdots B_{N-1}}^{\alpha} & \\
\geqslant & \left(2^{\frac{\alpha}{2}}-1\right) C_{A B_{m+1}}^{\alpha}+C_{A \mid B_{m+2} \cdots B_{N-1}}^{\alpha} \\
\geqslant & \left(2^{\frac{\alpha}{2}}-1\right)\left(C_{A B_{m+1}}^{\alpha}+\cdots+C_{A B_{N-2}}^{\alpha}\right) \\
& \quad+C_{A B_{N-1}}^{\alpha}
\end{aligned}
$$

Combining (6) and (7), we have Theorem 1. 
As for $\alpha \geqslant 2,\left(2^{\frac{\alpha}{2}}-1\right)^{m} \geqslant(\alpha / 2)^{m}$ for all $1 \leq m \leq N-3$, our formula (5D) in Theorem 1 gives a tighter monogamy relation with larger lower bounds than (11), (2). In Theorem 1, we have assumed that some $C_{A B_{i}} \geqslant C_{A \mid B_{i+1} \cdots B_{N-1}}$ and some $C_{A B_{j}} \leq C_{A \mid B_{j+1} \cdots B_{N-1}}$ for the $2 \otimes 2 \otimes \cdots \otimes 2$ mixed state $\rho \in \mathbb{H}_{A} \otimes \mathbb{H}_{B_{1}} \otimes$ $\cdots \otimes \mathbb{H}_{B_{N-1}}$. If all $C_{A B_{i}} \geqslant C_{A \mid B_{i+1} \cdots B_{N-1}}$ for $i=1,2, \cdots, N-2$, then we have the following conclusion:

Theorem 2. If $C_{A B_{i}} \geqslant C_{A \mid B_{i+1} \cdots B_{N-1}}$ for all $i=1,2, \cdots, N-2$, then we have

$$
\begin{aligned}
C_{A \mid B_{1} \cdots B_{N-1}}^{\alpha} & \\
\geqslant & C_{A B_{1}}^{\alpha}+\left(2^{\frac{\alpha}{2}}-1\right) C_{A B_{2}}^{\alpha}+\cdots+\left(2^{\frac{\alpha}{2}}-1\right)^{N-3} C_{A B_{N-2}}^{\alpha} \\
& +\left(2^{\frac{\alpha}{2}}-1\right)^{N-2} C_{A B_{N-1}}^{\alpha} .
\end{aligned}
$$

Example 1. Let us consider the three-qubit state $|\psi\rangle$ in the generalized Schmidt decomposition form [38, 39],

$$
\begin{aligned}
|\psi\rangle= & \lambda_{0}|000\rangle+\lambda_{1} e^{i \varphi}|100\rangle+\lambda_{2}|101\rangle \\
& +\lambda_{3}|110\rangle+\lambda_{4}|111\rangle,
\end{aligned}
$$

where $\lambda_{i} \geqslant 0, i=0,1,2,3,4$ and $\sum_{i=0}^{4} \lambda_{i}^{2}=1$. From the definition of concurrence, we have $C_{A \mid B C}=2 \lambda_{0} \sqrt{\lambda_{2}^{2}+\lambda_{3}^{2}+\lambda_{4}^{2}}, C_{A B}=2 \lambda_{0} \lambda_{2}$, and $C_{A C}=2 \lambda_{0} \lambda_{3}$. Set $\lambda_{0}=\lambda_{1}=\frac{1}{2}, \lambda_{2}=\lambda_{3}=\lambda_{4}=\frac{\sqrt{6}}{6}$, one has $C_{A \mid B C}=\frac{\sqrt{2}}{2}, C_{A B}=C_{A C}=\frac{\sqrt{6}}{6}$, then $C_{A \mid B C}^{\alpha}=\left(\frac{\sqrt{2}}{2}\right)^{\alpha}, C_{A B}^{\alpha}+C_{A C}^{\alpha}=2\left(\frac{\sqrt{6}}{6}\right)^{\alpha}, C_{A B}^{\alpha}+\frac{\alpha}{2} C_{A C}^{\alpha}=\left(1+\frac{\alpha}{2}\right)\left(\frac{\sqrt{6}}{6}\right)^{\alpha}$, $C_{A B}^{\alpha}+\left(2^{\frac{\alpha}{2}}-1\right) C_{A C}^{\alpha}=2^{\frac{\alpha}{2}}\left(\frac{\sqrt{6}}{6}\right)^{\alpha}$. One can see that our result is better than the results in 34] and [35] for $\alpha \geqslant 2$; see Fig 1.

\section{TIGHTER MONOGAMY REALATIONS FOR EoF}

The entanglement of formation (EOF) [40, 41] is a well-defined important measure of entanglement for bipartite systems. Let $\mathbb{H}_{A}$ and $\mathbb{H}_{B}$ be $m$ - and $n$ dimensional $(m \leqslant n)$ vector spaces, respectively. The EOF of a pure state $|\psi\rangle \in \mathbb{H}_{A} \otimes \mathbb{H}_{B}$ is defined by

$$
E(|\psi\rangle)=S\left(\rho_{A}\right),
$$

where $\rho_{A}=\operatorname{Tr}_{B}(|\psi\rangle\langle\psi|)$ and $S(\rho)=-\operatorname{Tr}\left(\rho \log _{2} \rho\right)$. For a bipartite mixed state $\rho_{A B} \in \mathbb{H}_{A} \otimes \mathbb{H}_{B}$, the entanglement of formation is given by

$$
E\left(\rho_{A B}\right)=\min _{\left\{p_{i},\left|\psi_{i}\right\rangle\right\}} \sum_{i} p_{i} E\left(\left|\psi_{i}\right\rangle\right)
$$

with the minimum taking over all possible pure-state decompositions of $\rho_{A B}$. 


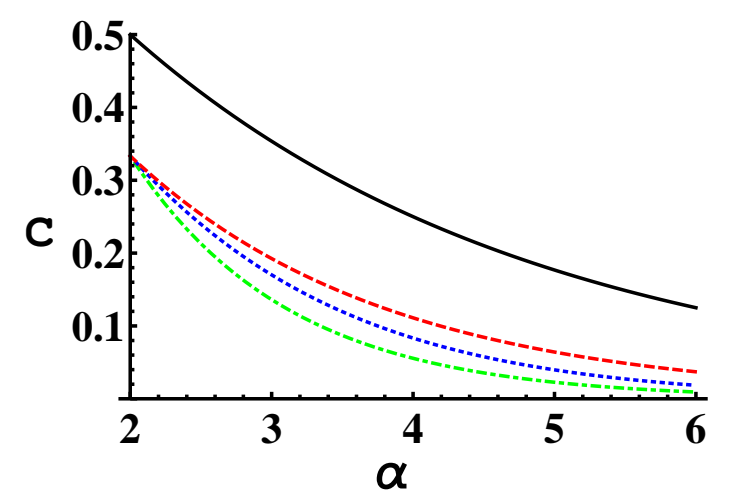

Figure 1: Behavior of the concurrence of $|\psi\rangle$ and its lower bound, which are functions of $\alpha$ plotted. The black solid line represents the concurrence of $|\psi\rangle$ in Example 1, the red dashed line represents the lower bound from our result, and the blue dotted (green dot-dashed) line represents the lower bound from the result in 35] (34]).

Denote $f(x)=H\left(\frac{1+\sqrt{1-x}}{2}\right)$, where $H(x)=-x \log _{2}(x)-(1-x) \log _{2}(1-x)$. From (10) and (11), one has $E(|\psi\rangle)=f\left(C^{2}(|\psi\rangle)\right)$ for $2 \otimes m(m \geqslant 2)$ pure state $|\psi\rangle$, and $E(\rho)=f\left(C^{2}(\rho)\right)$ for two-qubit mixed state $\rho$ [42]. It is obvious that $f(x)$ is a monotonically increasing function for $0 \leqslant x \leqslant 1 . f(x)$ satisfies the following relations:

$$
f^{\sqrt{2}}\left(x^{2}+y^{2}\right) \geqslant f^{\sqrt{2}}\left(x^{2}\right)+f^{\sqrt{2}}\left(y^{2}\right),
$$

where $f^{\sqrt{2}}\left(x^{2}+y^{2}\right)=\left[f\left(x^{2}+y^{2}\right)\right]^{\sqrt{2}}$.

It has been shown that the EOF does not satisfy the inequality $E_{A B}+E_{A C} \leq$ $E_{A \mid B C}$ [43]. In [44, the authors showed that EOF is a monotonic function satisfying $E^{2}\left(C_{A \mid B_{1} B_{2} \cdots B_{N-1}}^{2}\right) \geqslant E^{2}\left(\sum_{i=1}^{N-1} C_{A B_{i}}^{2}\right)$. For $N$-qubit systems, one has 34 .

$$
E_{A \mid B_{1} B_{2} \cdots B_{N-1}}^{\alpha} \geqslant E_{A B_{1}}^{\alpha}+E_{A B_{2}}^{\alpha}+\cdots+E_{A B_{N-1}}^{\alpha}
$$

for $\alpha \geqslant \sqrt{2}$, where $E_{A \mid B_{1} B_{2} \cdots B_{N-1}}$ is the entanglement of formation of $\rho$ in bipartite partition $A \mid B_{1} B_{2} \cdots B_{N-1}$, and $E_{A B_{i}}, i=1,2, \cdots, N-1$, is the EOF of the mixed states $\rho_{A B_{i}}=\operatorname{Tr}_{B_{1} B_{2} \cdots B_{i-1}, B_{i+1} \cdots B_{N-1}}(\rho)$. It is further proved that for $\alpha \geqslant \sqrt{2}$, if $C_{A B_{i}} \geqslant C_{A \mid B_{i+1} \cdots B_{N-1}}$ for $i=1,2, \cdots, m$ and $C_{A B_{j}} \leqslant$ $C_{A \mid B_{j+1} \cdots B_{N-1}}$ for $j=m+1, \cdots, N-2, \forall 1 \leqslant m \leqslant N-3, N \geqslant 4$, then [35.

$$
\begin{aligned}
E_{A \mid B_{1} B_{2} \cdots B_{N-1}}^{\alpha} & \\
\geqslant & E_{A B_{1}}^{\alpha}+(\alpha / \sqrt{2}) E_{A B_{2}}^{\alpha} \cdots+(\alpha / \sqrt{2})^{m-1} E_{A B_{m}}^{\alpha} \\
& +(\alpha / \sqrt{2})^{m+1}\left(E_{A B_{m+1}}^{\alpha}+\cdots+E_{A B_{N-2}}^{\alpha}\right) \\
& +(\alpha / \sqrt{2})^{m} E_{A B_{N-1}}^{\alpha},
\end{aligned}
$$


In fact, generally we can prove the following results.

Theorem 3. For any $N$-qubit mixed state $\rho \in \mathbb{H}_{A} \otimes \mathbb{H}_{B_{1}} \otimes \cdots \otimes \mathbb{H}_{B_{N-1}}$, if $C_{A B_{i}} \geqslant C_{A \mid B_{i+1} \cdots B_{N-1}}$ for $i=1,2, \cdots, m$, and $C_{A B_{j}} \leqslant C_{A \mid B_{j+1} \cdots B_{N-1}}$ for $j=m+1, \cdots, N-2, \forall 1 \leqslant m \leqslant N-3, N \geqslant 4$, the entanglement of formation $E(\rho)$ satisfies

$$
\begin{aligned}
E_{A \mid B_{1} B_{2} \cdots B_{N-1}}^{\alpha} & \\
\geqslant & E_{A B_{1}}^{\alpha}+\left(2^{t}-1\right) E_{A B_{2}}^{\alpha} \cdots+\left(2^{t}-1\right)^{m-1} E_{A B_{m}}^{\alpha} \\
& +\left(2^{t}-1\right)^{m+1}\left(E_{A B_{m+1}}^{\alpha}+\cdots+E_{A B_{N-2}}^{\alpha}\right) \\
& +\left(2^{t}-1\right)^{m} E_{A B_{N-1}}^{\alpha},
\end{aligned}
$$

for $\alpha \geqslant \sqrt{2}$, where $t=\alpha / \sqrt{2}$.

Proof. For $\alpha \geqslant \sqrt{2}$, we have

$$
\begin{aligned}
f^{\alpha}\left(x^{2}+y^{2}\right) & =\left(f^{\sqrt{2}}\left(x^{2}+y^{2}\right)\right)^{t} \\
& \geqslant\left(f^{\sqrt{2}}\left(x^{2}\right)+f^{\sqrt{2}}\left(y^{2}\right)\right)^{t} \\
& \geqslant\left(f^{\sqrt{2}}\left(x^{2}\right)\right)^{t}+\left(2^{t}-1\right)\left(f^{\sqrt{2}}\left(y^{2}\right)\right)^{t} \\
& =f^{\alpha}\left(x^{2}\right)+\left(2^{t}-1\right) f^{\alpha}\left(y^{2}\right),
\end{aligned}
$$

where the first inequality is due to the inequality (12), and the second inequality is obtained from a similar consideration in the proof of the second inequality in (4).

Let $\rho=\sum_{i} p_{i}\left|\psi_{i}\right\rangle\left\langle\psi_{i}\right| \in \mathbb{H}_{A} \otimes \mathbb{H}_{B_{1}} \otimes \cdots \otimes \mathbb{H}_{B_{N}-1}$ be the optimal decomposition of $E_{A \mid B_{1} B_{2} \cdots B_{N-1}}(\rho)$ for the $N$-qubit mixed state $\rho$; then we have

$$
\begin{aligned}
E_{A \mid B_{1} B_{2} \cdots B_{N-1}}(\rho) & =\sum_{i} p_{i} E_{A \mid B_{1} B_{2} \cdots B_{N-1}}\left(\left|\psi_{i}\right\rangle\right) \\
& =\sum_{i} p_{i} f\left(C_{A \mid B_{1} B_{2} \cdots B_{N-1}}^{2}\left(\left|\psi_{i}\right\rangle\right)\right) \\
& \geqslant f\left(\sum_{i} p_{i} C_{A \mid B_{1} B_{2} \cdots B_{N-1}}^{2}\left(\left|\psi_{i}\right\rangle\right)\right) \\
& \geqslant f\left(\left[\sum_{i} p_{i} C_{A \mid B_{1} B_{2} \cdots B_{N-1}}\left(\left|\psi_{i}\right\rangle\right)\right]^{2}\right) \\
& \geqslant f\left(C_{A \mid B_{1} B_{2} \cdots B_{N-1}}^{2}(\rho)\right)
\end{aligned}
$$

where the first inequality is due to the fact that $f(x)$ is a convex function. The second inequality is due to the Cauchy-Schwarz inequality: $\left(\sum_{i} x_{i}^{2}\right)^{\frac{1}{2}}\left(\sum_{i} y_{i}^{2}\right)^{\frac{1}{2}} \geqslant$ $\sum_{i} x_{i} y_{i}$, with $x_{i}=\sqrt{p_{i}}$ and $y_{i}=\sqrt{p_{i}} C_{A \mid B_{1} B_{2} \cdots B_{N-1}}\left(\left|\psi_{i}\right\rangle\right)$. Due to the definition 
of concurrence and that $f(x)$ is a monotonically increasing function, we obtain the third inequality. Therefore, we have

$$
\begin{aligned}
& E_{A \mid B_{1} B_{2} \cdots B_{N-1}}^{\alpha}(\rho) \\
& \geqslant f^{\alpha}\left(C_{A B_{1}}^{2}+C_{A B_{2}}^{2}+\cdots+C_{A B_{m-1}}^{2}\right) \\
& \geqslant f^{\alpha}\left(C_{A B_{1}}^{2}\right)+\left(2^{t}-1\right) f^{\alpha}\left(C_{A B_{2}}^{2}\right)+\cdots+\left(2^{t}-1\right)^{m-1} f^{\alpha}\left(C_{A B_{m}}^{2}\right) \\
&+\left(2^{t}-1\right)^{m+1}\left(f^{\alpha}\left(C_{A B_{m+1}}^{2}\right)+\cdots+f^{\alpha}\left(C_{A B_{N-2}}^{2}\right)\right) \\
&+\left(2^{t}-1\right)^{m} f^{\alpha}\left(C_{A B_{N-1}}^{2}\right) \\
&= E_{A \mid B_{1}}^{\alpha}+\left(2^{t}-1\right) E_{A B_{2}}^{\alpha}+\cdots+\left(2^{t}-1\right)^{m-1} E_{A B_{m}}^{\alpha} \\
&+\left(2^{t}-1\right)^{m+1}\left(E_{A B_{m+1}}^{\alpha}+\cdots+E_{A B_{N-2}}^{\alpha}\right) \\
&+\left(2^{t}-1\right)^{m} E_{A B_{N-1}}^{\alpha},
\end{aligned}
$$

where we have used the monogamy inequality in (1) for $N$-qubit states $\rho$ to obtain the first inequality. By using (16) and the similar consideration in the proof of Theorem 1, we get the second inequality. Since for any $2 \otimes$ 2 quantum state $\rho_{A B_{i}}, E\left(\rho_{A B_{i}}\right)=f\left[C^{2}\left(\rho_{A B_{i}}\right)\right]$, one gets the last equality.

As for $\left(2^{\alpha / \sqrt{2}}-1\right) \geqslant \alpha / \sqrt{2}$ for $\alpha \geqslant \sqrt{2}$, (15) is obviously tighter than (13), (14). Moreover, similar to the concurrence, for the case that $C_{A B_{i}} \geqslant$ $C_{A \mid B_{i+1} \cdots B_{N-1}}$ for all $i=1,2, \cdots, N-2$, we have a simple tighter monogamy relation for the entanglement of formation:

Theorem4. If $C_{A B_{i}} \geqslant C_{A \mid B_{i+1} \cdots B_{N-1}}$ for all $i=1,2, \cdots, N-2$, we have

$$
\begin{aligned}
E_{A \mid B_{1} B_{2} \cdots B_{N-1}}^{\alpha} \quad \geqslant & E_{A B_{1}}^{\alpha}+\left(2^{\alpha / \sqrt{2}}-1\right) E_{A B_{2}}^{\alpha}+\cdots \\
& +\left(2^{\alpha / \sqrt{2}}-1\right)^{N-2} E_{A B_{N-1}}^{\alpha}
\end{aligned}
$$

for $\alpha \geqslant \sqrt{2}$.

Example 2. Let us consider the $W$ state, $|W\rangle=\frac{1}{\sqrt{3}}(|100\rangle+|010\rangle+|001\rangle)$. We have $E_{A B}=E_{A C}=0.550048, E_{A \mid B C}=0.918296$, and then $E_{A \mid B C}^{\alpha}=$ $(0.918296)^{\alpha}, E_{A B}^{\alpha}+E_{A C}^{\alpha}=2(0.550048)^{\alpha}, E_{A B}^{\alpha}+\frac{\alpha}{\sqrt{2}} E_{A C}^{\alpha}=\left(1+\frac{\alpha}{\sqrt{2}}\right)(0.550048)^{\alpha}$, $E_{A B}^{\alpha}+\left(2^{\frac{\alpha}{\sqrt{2}}}-1\right) E_{A C}^{\alpha}=2^{\frac{\alpha}{\sqrt{2}}}(0.550048)^{\alpha}$. It is easily verified that our results are better than the results in 34 and 35 ] for $\alpha \geqslant \sqrt{2}$; see Fig 2 .

\section{TIGHTER MONOGAMY RELATIONS FOR NEGATIVITY}

Another well-known quantifier of bipartite entanglement is the negativity. Given a bipartite state $\rho_{A B}$ in $\mathbb{H}_{A} \otimes \mathbb{H}_{B}$, the negativity is defined by [45] $N\left(\rho_{A B}\right)=$ $\left(\left\|\rho_{A B}^{T_{A}}\right\|-1\right) / 2$, where $\rho_{A B}^{T_{A}}$ is the partial transpose with respect to the subsystem $A$, and $\|X\|$ denotes the trace norm of $X$, i.e $\|X\|=\operatorname{Tr} \sqrt{X X^{\dagger}}$. Negativity is a computable measure of entanglement and is a convex function of $\rho_{A B}$. It vanishes if and only if $\rho_{A B}$ is separable for the $2 \otimes 2$ and $2 \otimes 3$ systems [46]. 


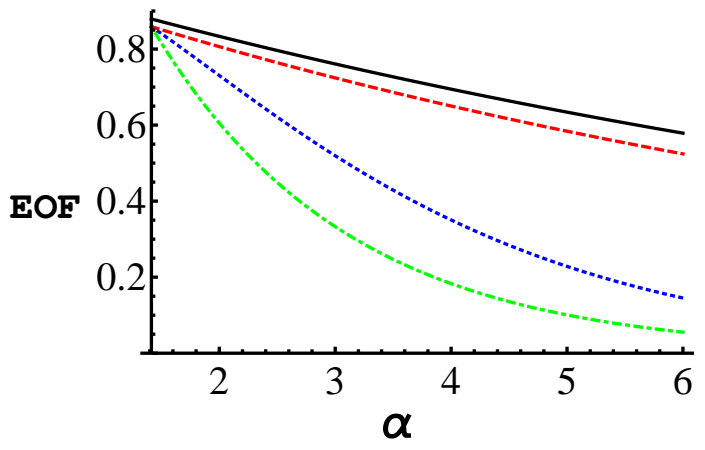

Figure 2: Behavior of the EOF of $|W\rangle$ and its lower bound, which are functions of $\alpha$ plotted. The black solid line represents the EOF of the state $|W\rangle$ in Example 2, the red dashed line represents the lower bound from our result, and the blue dotted (green dot-dashed) line represents the lower bound from the result in [35] (34]).

For the purpose of discussion, we use the following definition of negativity, $N\left(\rho_{A B}\right)=\left\|\rho_{A B}^{T_{A}}\right\|-1$. For any bipartite pure state $|\psi\rangle_{A B}$, the negativity $N\left(\rho_{A B}\right)$ is given by $N\left(|\psi\rangle_{A B}\right)=2 \sum_{i<j} \sqrt{\lambda_{i} \lambda_{j}}=\left(\operatorname{Tr} \sqrt{\rho_{A}}\right)^{2}-1$, where $\lambda_{i}$ are the eigenvalues for the reduced density matrix of $|\psi\rangle_{A B}$. For a mixed state $\rho_{A B}$, the convex-roof extended negativity (CREN) is defined as

$$
N_{c}\left(\rho_{A B}\right)=\min \sum_{i} p_{i} N\left(\left|\psi_{i}\right\rangle_{A B}\right)
$$

where the minimum is taken over all possible pure-state decompositions $\left\{p_{i},\left|\psi_{i}\right\rangle_{A B}\right\}$ of $\rho_{A B}$. CREN gives a perfect discrimination of positive partial transposed bound entangled states and separable states in any bipartite quantum system [47, 48.

Let us consider the relation between CREN and concurrence. For any bipartite pure state $|\psi\rangle_{A B}$ in a $d \otimes d$ quantum system with Schmidt rank 2, $|\psi\rangle_{A B}=\sqrt{\lambda_{0}}|00\rangle+\sqrt{\lambda_{1}}|11\rangle$, one has $N\left(|\psi\rangle_{A B}\right)=\||\psi\rangle\left\langle\left.\psi\right|^{T_{B}} \|-1=2 \sqrt{\lambda_{0} \lambda_{1}}=\right.$ $\sqrt{2\left(1-\operatorname{Tr} \rho_{A}^{2}\right)}=C\left(|\psi\rangle_{A B}\right)$. In other words, negativity is equivalent to concurrence for any pure state with Schmidt rank 2, and consequently it follows that for any two-qubit mixed state $\rho_{A B}=\sum p_{i}\left|\psi_{i}\right\rangle_{A B}\left\langle\psi_{i}\right|$,

$$
\begin{aligned}
N_{c}\left(\rho_{A B}\right) & =\min \sum_{i} p_{i} N\left(\left|\psi_{i}\right\rangle_{A B}\right) \\
& =\min \sum_{i} p_{i} C\left(\left|\psi_{i}\right\rangle_{A B}\right) \\
& =C\left(\rho_{A B}\right) .
\end{aligned}
$$

With a similar consideration of concurrence, we obtain the following result. 
Theorem 5. For any $N$-qubit state $\rho \in \mathbb{H}_{A} \otimes \mathbb{H}_{B_{1}} \otimes \cdots \otimes \mathbb{H}_{B_{N-1}}$, if $N_{c A B_{i}} \geqslant$ $N_{c A \mid B_{i+1} \cdots B_{N-1}}$ for $i=1,2, \cdots, m$, and $N_{c A B_{j}} \leqslant N_{c A \mid B_{j+1} \cdots B_{N-1}}$ for $j=m+$ $1, \cdots, N-2, \forall 1 \leqslant m \leqslant N-3, N \geqslant 4$, we have

$$
\begin{aligned}
N_{c A \mid B_{1} B_{2} \cdots B_{N-1}}^{\alpha} & \\
\geqslant & N_{c A B_{1}}^{\alpha}+\left(2^{\frac{\alpha}{2}}-1\right) N_{c A B_{2}}^{\alpha}+\cdots+\left(2^{\frac{\alpha}{2}}-1\right)^{m-1} N_{c A B_{m}}^{\alpha} \\
& +\left(2^{\frac{\alpha}{2}}-1\right)^{m+1}\left(N_{c A B_{m+1}}^{\alpha}+\cdots+N_{c A B_{N-2}}^{\alpha}\right) \\
& +\left(2^{\frac{\alpha}{2}}-1\right)^{m} N_{c A B_{N-1}}^{\alpha}
\end{aligned}
$$

for all $\alpha \geqslant 2$.

In Theorem 5 we have assumed that some $N_{c A B_{i}} \geqslant N_{c A \mid B_{i+1} \cdots B_{N-1}}$ and some $N_{c A B_{j}} \leq N_{c A \mid B_{j+1} \cdots B_{N-1}}$ for the $2 \otimes 2 \otimes \cdots \otimes 2$ mixed state $\rho \in \mathbb{H}_{A} \otimes$ $\mathbb{H}_{B_{1}} \otimes \cdots \otimes \mathbb{H}_{B_{N-1}}$. If all $N_{c A B_{i}} \geqslant N_{c A \mid B_{i+1} \cdots B_{N-1}}$ for $i=1,2, \cdots, N-2$, then we have the following conclusion:

Theorem 6. If $N_{c A B_{i}} \geqslant N_{c A \mid B_{i+1} \cdots B_{N-1}}$ for all $i=1,2, \cdots, N-2$, then we have

$$
\begin{aligned}
N_{c A \mid B_{1} \cdots B_{N-1}}^{\alpha} \geqslant & N_{C A B_{1}}^{\alpha}+\left(2^{\frac{\alpha}{2}}-1\right) N_{c A B_{2}}^{\alpha}+\cdots \\
& +\left(2^{\frac{\alpha}{2}}-1\right)^{N-2} N_{C A B_{N-1}}^{\alpha} .
\end{aligned}
$$

Example 3. Let us consider again the three-qubit state $|\psi\rangle$ (9). From the definition of CREN, we have $N_{c A \mid B C}=2 \lambda_{0} \sqrt{\lambda_{2}^{2}+\lambda_{3}^{2}+\lambda_{4}^{2}}, N_{c A B}=2 \lambda_{0} \lambda_{2}$, and $N_{c A C}=2 \lambda_{0} \lambda_{3}$. Set $\lambda_{0}=\lambda_{1}=\lambda_{2}=\lambda_{3}=\lambda_{4}=\frac{\sqrt{5}}{5}$. One gets $N_{c A \mid B C}^{\alpha}=\left(\frac{2 \sqrt{3}}{5}\right)^{\alpha}$, $N_{c A B}^{\alpha}+N_{c A C}^{\alpha}=2\left(\frac{2}{5}\right)^{\alpha}, N_{c A B}^{\alpha}+\frac{\alpha}{2} N_{c A C}^{\alpha}=\left(1+\frac{\alpha}{2}\right)\left(\frac{2}{5}\right)^{\alpha}, N_{c A B}^{\alpha}+\left(2^{\frac{\alpha}{2}}-1\right) N_{c A C}^{\alpha}=$ $2^{\frac{\alpha}{2}}\left(\frac{2}{5}\right)^{\alpha}$. One can see that our result is better than the results in 34 and [36] for $\alpha \geqslant 2$; see Fig. 3 .

\section{Tighter monogamy relations for Tsallis-q en- tanglement}

For a bipartite pure state $|\psi\rangle_{A B}$, the Tsallis- $q$ entanglement is defined by 24 .

$$
T_{q}\left(|\psi\rangle_{A B}\right)=S_{q}\left(\rho_{A}\right)=\frac{1}{q-1}\left(1-\operatorname{tr} \rho_{A}^{q}\right),
$$

for any $q>0$ and $q \neq 1$. If $q$ tends to $1, T_{q}(\rho)$ converges to the von Neumann entropy, $\lim _{q \rightarrow 1} T_{q}(\rho)=-\operatorname{tr} \rho \ln \rho=S_{q}(\rho)$. For a bipartite mixed state $\rho_{A B}$, Tsallis- $q$ entanglement is defined via the convex-roof extension, $T_{q}\left(\rho_{A B}\right)=$ $\min \sum_{i} p_{i} T_{q}\left(\left|\psi_{i}\right\rangle_{A B}\right)$, with the minimum taken over all possible pure-state decompositions of $\rho_{A B}$.

In [4, the author has proved an analytic relationship between Tsallis- $q$ entanglement and concurrence for $\frac{5-\sqrt{13}}{2} \leq q \leq \frac{5+\sqrt{13}}{2}$,

$$
T_{q}\left(|\psi\rangle_{A B}\right)=g_{q}\left(C^{2}\left(|\psi\rangle_{A B}\right)\right),
$$




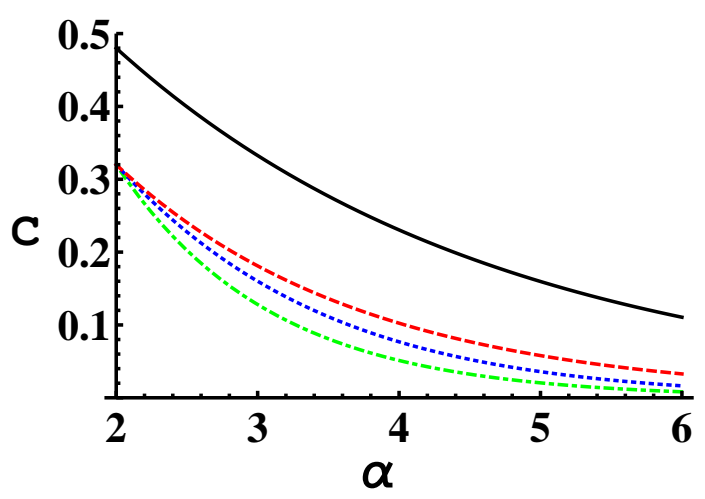

Figure 3: Behavior of the concurrence of $|\psi\rangle$ and its lower bound, which are functions of $\alpha$ plotted. The black solid line represents the concurrence of $|\psi\rangle$ in Example 3, the red dashed line represents the lower bound from our result, and the blue dotted (green dot-dashed) line represents the lower bound from the result in [36] $(34)$.

where the function $g_{q}(x)$ is defined as

$$
g_{q}(x)=\frac{1}{q-1}\left[1-\left(\frac{1+\sqrt{1-x}}{2}\right)^{q}-\left(\frac{1-\sqrt{1-x}}{2}\right)^{q}\right] .
$$

It has been shown that $T_{q}(|\psi\rangle)=g_{q}\left(C^{2}(|\psi\rangle)\right)$ for $2 \otimes m(m \geqslant 2)$ pure state $|\psi\rangle$, and $T_{q}(\rho)=g_{q}\left(C^{2}(\rho)\right)$ for two-qubit mixed state $\rho$ in [24]. Hence, (23) holds for any $q$ such that $g_{q}(x)$ in (24) is monotonically increasing and convex. In particular, $g_{q}(x)$ satisfies the following relations for $2 \leqslant q \leqslant 3$ :

$$
g_{q}\left(x^{2}+y^{2}\right) \geqslant g_{q}\left(x^{2}\right)+g_{q}^{2}\left(y^{2}\right) .
$$

The Tsallis- $q$ entanglement satisfies 24]

$$
T_{q_{A \mid B_{1} B_{2} \cdots B_{N-1}}} \geqslant \sum_{i=1}^{N-1} T_{q_{A B_{i}}},
$$

where $i=1,2, \cdots N-1,2 \leqslant q \leqslant 3$. It is further proved in [49]

$$
T_{q A \mid B_{1} B_{2} \cdots B_{N-1}}^{2} \geqslant \sum_{i=1}^{N-1} T_{q A B_{i}}^{2},
$$

with $\frac{5-\sqrt{13}}{2} \leqslant q \leqslant \frac{5+\sqrt{13}}{2}$. In fact, generally we can prove the following results.

Theorem 7. For an arbitrary $N$-qubit mixed state $\rho_{A B_{1} \cdots B_{N-1}}$, if $C_{A B_{i}} \geqslant$ $C_{A \mid B_{i+1} \cdots B_{N-1}}$ for $i=1,2, \cdots, m$, and $C_{A B_{j}} \leqslant C_{A \mid B_{j+1} \cdots B_{N-1}}$ for $j=m+$ $1, \cdots, N-2, \forall 1 \leqslant m \leqslant N-3, N \geqslant 4$, then the $\alpha$ th power of Tsallis- $q$ satisfies 
the monogamy relation

$$
\begin{aligned}
& T_{q_{A \mid B_{1} B_{2} \cdots B_{N-1}}^{\alpha}} \\
& \geqslant \\
& \quad T_{q_{A B_{1}}}^{\alpha}+\left(2^{\alpha}-1\right) T_{q_{A B_{2}}}^{\alpha}+\cdots+\left(2^{\alpha}-1\right)^{m-1} T_{q_{A B_{m}}}^{\alpha} \\
& \quad+\left(2^{\alpha}-1\right)^{m+1}\left(T_{q_{A B_{m+1}}^{\alpha}}^{\alpha}+\cdots+T_{q_{A B_{N-2}}^{\alpha}}^{\alpha}\right) \\
& \quad+\left(2^{\alpha}-1\right)^{m} T_{q_{A B_{N-1}}}^{\alpha},
\end{aligned}
$$

where $\alpha \geqslant 1, T_{q A \mid B_{1} B_{2} \cdots B_{N-1}}$ quantifies the Tsallis- $q$ entanglement in the partition $A \mid B_{1} B_{2} \cdots B_{N-1}$ and $T_{q_{A B_{i}}}$ quantifies that in two-qubit subsystem $A B_{i}$ with $2 \leqslant q \leqslant 3$.

Proof. For $\alpha \geqslant 1$, we have

$$
\begin{aligned}
g_{q}^{\alpha}\left(x^{2}+y^{2}\right) & \geqslant\left(g_{q}\left(x^{2}\right)+g_{q}\left(y^{2}\right)\right)^{\alpha} \\
& \geqslant g_{q}^{\alpha}\left(x^{2}\right)+\left(2^{\alpha}-1\right) g_{q}^{\alpha}\left(y^{2}\right),
\end{aligned}
$$

where the first inequality is due to the inequality (25), and the second inequality is obtained from a similar consideration in the proof of the second inequality in (44).

Let $\rho=\sum_{i} p_{i}\left|\psi_{i}\right\rangle\left\langle\psi_{i}\right| \in \mathbb{H}_{A} \otimes \mathbb{H}_{B_{1}} \otimes \cdots \otimes \mathbb{H}_{B_{N}-1}$ be the optimal decomposition for the $N$-qubit mixed state $\rho$; then we have

$$
\begin{aligned}
& T_{q A \mid B_{1} B_{2} \cdots B_{N-1}}(\rho) \\
& =\sum_{i} p_{i} T_{q}\left(\left|\psi_{i}\right\rangle_{A \mid B_{1} B_{2} \cdots B_{N-1}}\right) \\
& =\sum_{i} p_{i} g_{q}\left[C_{A \mid B_{1} B_{2} \cdots B_{N-1}}^{2}\left(\left|\psi_{i}\right\rangle\right)\right] \\
& \geqslant g_{q}\left[\sum_{i} p_{i} C_{A \mid B_{1} B_{2} \cdots B_{N-1}}^{2}\left(\left|\psi_{i}\right\rangle\right)\right] \\
& \geqslant g_{q}\left(\left[\sum_{i} p_{i} C_{A \mid B_{1} B_{2} \cdots B_{N-1}}\left(\left|\psi_{i}\right\rangle\right)\right]^{2}\right) \\
& =g_{q}\left[C_{A \mid B_{1} B_{2} \cdots B_{N-1}}^{2}(\rho)\right],
\end{aligned}
$$

where the first inequality is due to the fact that $g_{q}(x)$ is a convex function. The second inequality is due to the Cauchy-Schwarz inequality: $\left(\sum_{i} x_{i}^{2}\right)^{\frac{1}{2}}\left(\sum_{i} y_{i}^{2}\right)^{\frac{1}{2}} \geqslant$ $\sum_{i} x_{i} y_{i}$, with $x_{i}=\sqrt{p_{i}}$ and $y_{i}=\sqrt{p_{i}} C_{A \mid B_{1} B_{2} \cdots B_{N-1}}\left(\left|\psi_{i}\right\rangle\right)$. Due to the definition of Tsallis- $q$ entanglement and that $g_{q}(x)$ is a monotonically increasing function, 


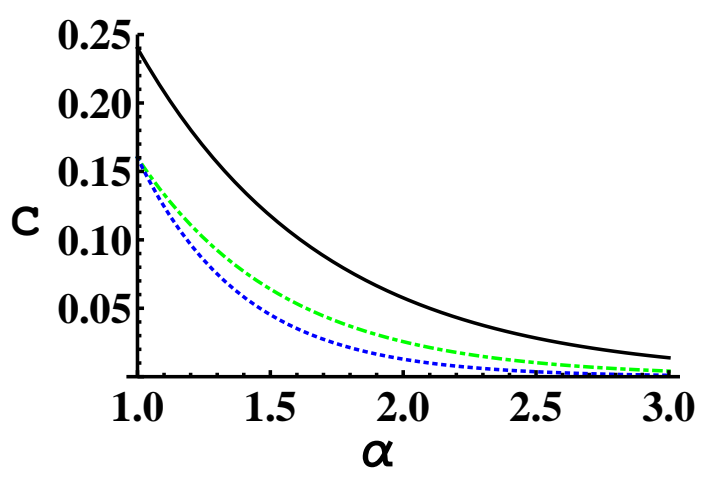

Figure 4: Behavior of the concurrence of $|\psi\rangle$ and its lower bound, which are functions of $\alpha$ plotted. The black solid line represents the concurrence of $|\psi\rangle$ in Example 4, the green dot-dashed line represents the lower bound from our result, and the blue dotted line represents the lower bound from the result in 24].

we obtain the third inequality. Therefore, we have

$$
\begin{aligned}
& T_{q A \mid B_{1} B_{2} \cdots B_{N-1}}^{\alpha}(\rho) \\
& \geqslant g_{q}^{\alpha}\left[\sum_{i} C^{2}\left(\rho_{A B_{i}}\right)\right] \\
& \geqslant g_{q}{ }^{\alpha}\left(C_{A B_{1}}\right)+\left(2^{\alpha}-1\right) g_{q}{ }^{\alpha}\left(C_{A B_{2}}\right)+\cdots \\
&+\left(2^{\alpha}-1\right)^{m-1} g_{q}{ }^{\alpha}\left(C_{A B_{m}}\right) \\
&+\left(2^{\alpha}-1\right)^{m+1}\left(g_{q}{ }^{\alpha}\left(C_{A B_{m+1}}\right)+\cdots+g_{q}{ }^{\alpha}\left(C_{A B_{N-2}}\right)\right) \\
&+\left(2^{\alpha}-1\right)^{m} g_{q}{ }^{\alpha}\left(C_{A B_{N-1}}\right) \\
&= T_{q A B_{1}}^{\alpha}+\left(2^{\alpha}-1\right) T_{q A B_{2}}+\cdots+\left(2^{\alpha}-1\right)^{m-1} T_{q A B_{m}}{ }^{\alpha} \\
&+\left(2^{\alpha}-1\right)^{m+1}\left(T_{q_{A B_{m+1}}}^{\alpha}+\cdots+T_{q_{A B_{N-2}}}{ }^{\alpha}\right. \\
&+\left(2^{\alpha}-1\right)^{m} T_{q_{A B_{N-1}}},
\end{aligned}
$$

where we have used the monogamy inequality in (1) for $N$-qubit states $\rho$ to obtain the first inequality. By using (29) and the similar consideration in the proof of Theorem 1, we get the second inequality. Since for any $2 \otimes 2$ quantum state $\rho_{A B_{i}}, T_{q}\left(\rho_{A B_{i}}\right)=g_{q}\left[C^{2}\left(\rho_{A B_{i}}\right)\right]$, one gets the last equality.

Example 4. Let us consider again the three-qubit state $|\psi\rangle(9)$. From the definition of Tsallis- $q$ entanglement, when $q=2$, we have $T_{2 A \mid B C}=2 \lambda_{0}^{2}\left(\lambda_{2}^{2}+\right.$ $\left.\lambda_{3}^{2}+\lambda_{4}^{2}\right), T_{2 A B}=2 \lambda_{0}^{2} \lambda_{2}^{2}$, and $T_{2 A C}=2 \lambda_{0}^{2} \lambda_{3}^{2}$. Set $\lambda_{0}=\lambda_{1}=\lambda_{2}=\lambda_{3}=\lambda_{4}=\frac{\sqrt{5}}{5}$. One gets $T_{2 A \mid B C}^{\alpha}=\left(\frac{6}{25}\right)^{\alpha}, T_{2 A B}^{\alpha}+T_{2}^{\alpha}{ }_{A C}^{\alpha}=2\left(\frac{2}{25}\right)^{\alpha}, T_{2}^{\alpha}{ }_{A B}+\left(2^{\frac{\alpha}{2}}-1\right) T_{2 A C}^{\alpha}=$ $2^{\alpha}\left(\frac{2}{25}\right)^{\alpha}$. One can see that our result is better than that in 34 for $\alpha \geqslant 2$; see Fig. 4 . 


\section{6 conclusion}

Entanglement monogamy is a fundamental property of multipartite entangled states. We have presented monogamy relations related to the $\alpha$ power of concurrence $C$, entanglement of formation $E$, negativity $N_{c}$, and Tsallis- $q$ entanglement $T_{q}$, which are tighter, at least for some classes of quantum states, than the existing entanglement monogamy relations for $\alpha>2, \alpha>\sqrt{2}, \alpha>2, \alpha>1$, respectively. The necessary conditions that our inequalities are strictly tighter can been seen from our monogamy relations. For instance, (8) s tighter than the existing ones for $\alpha>2$, for all quantum states where at least one of the $C_{A B_{i}}$ 's $(i=2, \cdots, N-1)$ is not zero, which excludes the fully separable states that have no entanglement distribution at all among the subsystems. Another case that $C_{A B_{i}}=0$ for all $i=2, \cdots, N-1$ is the $N$-qubit GHZ state [50], which is genuine multipartite entangled. However, for the genuine entangled $N$-qubit $W$ state [51, one has $C_{A B_{i}}=\frac{2}{N}, i=2, \cdots, N-1$, In general, most of states have at least one nonzero $C_{A B_{i}}(i=2, \cdots, N-1)$.

Monogamy relations characterize the distributions of entanglement in multipartite systems. Tighter monogamy relations imply finer characterizations of the entanglement distribution. Our approach may also be used to further study the monogamy properties related to other quantum correlations.

Acknowledgments This work is supported by the NSF of China under Grant No. 11675113 and is supported by the Research Foundation for Youth Scholars of Beijing Technology and Business University QNJJ2017-03.

\section{References}

[1] M. A. Nielsen and I. L. Chuang, Quantum Computation and Quantum Information (Cambridge University Press, Cambridge, 2000).

[2] R. Horodecki, P. Horodecki, M. Horodecki, and K. Horodecki, Quantum entanglement, Rev. Mod. Phys. 81, 865 (2009).

[3] F. Mintert, M. Kuś, and A. Buchleitner, Concurrence of Mixed Bipartite Quantum States in Arbitrary Dimensions, Phys. Rev. Lett. 92, 167902 (2004).

[4] K. Chen, S. Albeverio, and S. M. Fei, Concurrence of Arbitrary Dimensional Bipartite Quantum States, Phys. Rev. Lett. 95, 040504 (2005).

[5] H. P. Breuer, Separability criteria and bounds for entanglement measures, J. Phys. A: Math. Gen. 39, 11847 (2006).

[6] H. P. Breuer, Optimal Entanglement Criterion for Mixed Quantum States, Phys. Rev. Lett. 97, 080501 (2006).

[7] J. I. de Vicente, Lower bounds on concurrence and separability conditions, Phys. Rev. A 75, 052320 (2007). 
[8] C. J. Zhang, Y. S. Zhang, S. Zhang, and G. C. Guo, Optimal entanglement witnesses based on local orthogonal observables, Phys. Rev. A 76, 012334 (2007).

[9] M. Pawlowski, Security proof for cryptographic protocols based only on the monogamy ofBells inequality violations, Phys.Rev. A 82, 032313 (2010).

[10] M. Koashi and A.Winter, Monogamy of quantum entanglement and other correlations, Phys. Rev. A 69, 022309 (2004).

[11] T. J. Osborne and F. Verstraete, General Monogamy Inequality for Bipartite Qubit Entanglement, Phys. Rev. Lett. 96, 220503 (2006).

[12] Y. K. Bai, M. Y. Ye, and Z. D. Wang, Entanglement monogamy and entanglement evolution in multipartite systems, Phys. Rev. A 80, 044301 (2009).

[13] T. R. de Oliveira,M. F. Cornelio, and F. F. Fanchini, Monogamy of entanglement of formation, Phys. Rev. A 89, 034303 (2014).

[14] G. Adesso and F. Illuminati, Continuous variable tangle, monogamy inequality, and entanglement sharing in Gaussian states of continuous variable systems, New J. Phys. 8, 15 (2006).

[15] T. Hiroshima, G. Adesso, and F. Illuminati, Monogamy Inequality for Distributed Gaussian Entanglement, Phys. Rev. Lett. 98, 050503 (2007).

[16] G. Adesso and F. Illuminati, Strong Monogamy of Bipartite and Genuine Multiparitie Entanglement: the Guussian Case, Phys. Rev. Lett. 99, 150501 (2007).

[17] M. Christandl and A. Winter, Squashed entanglement: An additive entanglement measure, J. Math. Phys. 45, 829 (2004).

[18] D. Yang et al., Squashed entanglement for multipartite states and entanglement measures based on the mixed convex roof, IEEE Trans. Inf. Theory 55,3375 (2009).

[19] Y. C. Ou andH. Fan,Monogamy inequality in terms of negativity for threequbit states, Phys. Rev. A 75, 062308 (2007).

[20] J. S. Kim, A. Das, and B. C. Sanders, Entanglement monogamy of multipartite higher-dimensional quantum systems using convex-roof extend negativity, Phys. Rev. A 79, 012329 (2009).

[21] H. He and G. Vidal, Disentangling theorem and monogamy for entanglement negativity, Phys. Rev. A 91, 012339 (2015).

[22] J. H. Choi and J. S. Kim, Negativity and strong monogamy of multiparty quantum entanglement beyond qubits, Phys. Rev. A 92, 042307 (2015). 
[23] Y. Luo and Y. Li, Monogamy of $\alpha$-th power entanglement measurement in qubit system, Ann. Phys. 362, 511 (2015).

[24] J. S. Kim, Tsallis entropy and entanglement constraints in multiqubit systems, Phys. Rev. A 81, 062328 (2010).

[25] J. S. Kim, Generalized entanglement constraints in multi-qubit systems in terms of Tsallis entropy, Ann. Phys. 373, 197 (2016).

[26] J. S. Kim and B. C. Sanders,Monogamy of multi-qubit entanglement using Rényi entropy, J. Phys. A: Math. Theor. 43, 445305 (2010).

[27] M. F. Cornelio andM. C. de Oliveira, Strong superadditivity and monogamy of the Renyi measure of entanglement, Phys. Rev. A 81, 032332 (2010).

[28] Y.-X. Wang, L.-Z. Mu, V. Vedral, and H. Fan, Entanglement ényi-entropy, Phys. Rev. A 93, 022324 (2016).

[29] A. Uhlmann, Fidelity and concurrence of conjugated states, Phys. Rev. A 62, 032307 (2000).

[30] P. Rungta, V. Buzek, C. M. Caves, M. Hillery, and G. J. Milburn, Universal state inversion and concurrence in arbitrary dimensions, Phys. Rev. A 64, 042315 (2001).

[31] S. Albeverio and S. M. Fei, A note on invariants and entanglements, J. Opt. B: Quantum Semiclass Opt. 3, 223 (2001).

[32] T. Laustsen, F. Verstraete, and S. J. Van Enk, Local vs. joint measurements for the entanglement of assistance, Quantum Inf. Comput. 3, 64 (2003).

[33] C. S. Yu and H. S. Song, Entanglement monogamy of tripartite quantum states, Phys. Rev. A 77, 032329 (2008).

[34] X. N. Zhu and S. M. Fei, Entanglement monogamy relations of qubit systems, Phys. Rev. A 90, 024304 (2014).

[35] Z. X. Jin and S. M. Fei, Tighter entanglement monogamy relations of qubit systems, Quantum Inf. Proc. 16, 77 (2017).

[36] Z. X. Jin and S. M. Fei, Tighter monogamy relations of quantum entanglement for multiqubit W-class states, Quantum Inf. Proc. 17, 2 (2018).

[37] X. J. Ren and W. Jiang, Entanglement monogamy inequality in a $2 \otimes 2 \otimes 4$ system, Phys. Rev. A 81, 024305 (2010).

[38] A. Acin, A. Andrianov, L. Costa, E. Jané, J. I. Latorre, and R. Tarrach, Generalized Schmidt Decomposition and Classification of Three-QuantumBit States, Phys. Rev. Lett. 85, 1560 (2000).

[39] X. H. Gao and S. M. Fei, Estimation of concurrence for multipartite mixed states, Eur. Phys. J. Spec. Topics 159, 71 (2008). 
[40] C. H. Bennett, H. J. Bernstein, S. Popescu, and B. Schumacher, Concentrating partial entanglement by local operations, Phys. Rev. A 53, 2046 (1996).

[41] C. H. Bennett, D. P. DiVincenzo, J. A. Smolin, andW. K.Wootters, Mixedstate entanglement and quantum error correction, Phys. Rev. A 54, 3824 (1996).

[42] W. K.Wootters,Entanglement of Formation of anArbitrary State of Two Qubits, Phys. Rev. Lett. 80, 2245 (1998).

[43] V. Coffman, J. Kundu, and W. K. Wootters, Distributed entanglement, Phys. Rev. A 61, 052306 (2000).

[44] Y. K. Bai, N. Zhang, M. Y. Ye, and Z. D. Wang, Exploring multipartite quantum correlations with the square of quantum discord, Phys. Rev. A 88, 012123 (2013).

[45] G. Vidal and R. F. Werner, Computable measure of entanglement, Phys. Rev. A. 65, 032314 (2002).

[46] M. Horodecki, P. Horodecki, and R. Horodecki, Mixed-State Entanglement and Distillation: Is there a Bound Entanglement in Nature ?, Phys. Rev. Lett. 80, 5239 (1998).

[47] P. Horodeki, Separability criterion and inseparable mixed states with positive partial transposition, Phys. Lett. A. 232, 333 (1997).

[48] W. Dür, J. I. Cirac, M. Lewenstein, and D. Bruß, Distillability and partial transposition in bipartite systems, Phys. Rev. A. 61, 062313 (2000).

[49] G. M. Yuan, W. Song, M. Yang, D. C. Li, J. L. Zhao, and Z. L. Cao, Monogamy relation of multi-qubit systems for squared Tsallis-q entanglement, Sci. Rep. 6, 28719 (2016).

[50] D. Bouwmeester, J. W. Pan, M. Daniell, H. Weinfurter, and A. Zeilinger, Observation of Three-Photon Greenberger-Horne- Zeilinger Entanglement, Phys. Rev. Lett. 82, 1345 (1999).

[51] X. N. Zhu and S. M. Fei, General monogamy relations of quantum entanglement for multiqubit W-class states, Quantum Inf. Proc. 16, 53 (2017). 